\title{
FETAL HEART RATE CLASSIFICATION AND COMPARATIVE ANALYSIS USING CARDIOTOCOGRAPHY DATA AND KNOWN CLASSIFIERS
}

\author{
Razman Afridi ${ }^{1}$, Zafar Iqbal ${ }^{2}$, Muzammil Khan ${ }^{3 *}$, Arshad Ahmad ${ }^{4}$ and \\ Rashid Naseem ${ }^{5}$ \\ Department of Computer Science, City University of Science and Information \\ Technology, Pakistan \\ 11razmn_afridi@yahoo.com, ${ }^{2}$ zafarkhattak@gmail.com, \\ ${ }^{3}$ muzammilkhan86@googlemail.com, ${ }^{4}$ yaarshad@gmail.com, \\ ${ }^{5}$ rashid@ cusit.edu.pk
}

\begin{abstract}
The problem of fetal distress usually become one of the major reason of complication during child delivery. Fetal heart rate (FHR) is one of the pivotal ways to identify the occurrence of fetal distress. Cardiotocography (CTG) is the most widely practiced technique to record FHR. Improper analysis of CTG's graph may lead to serious loss. This study presents six classification algorithms: Decision Tree (DT), K-Nearest Neighbors (KNN), Logistic Regression (LR), Support Vector Machine (SVM), Random Forest (RF) and Naïve Bayes (NB), used for the classification of CTG data. To improve the performance of the classifiers, a corelation based feature selection technique is employed over the dataset to remove the unnecessary attributes. The performance of the classification algorithms is evaluated using evaluation metrics: Accuracy, Precision, Recall, and F-measure. The results revealed that Naïve Bayes achieved $83.06 \%$ accuracy, $92.20 \%$ precision, $83.10 \%$ recall and $86.90 \%$ f-measure.
\end{abstract}

Keywords - Cardiotocography; Decision Tree; K-Nearest Neighbors; Logistic Regression; Support Vector Machine; Random Forest; Naïve Bayes

\section{INTRODUCTION}

The area of medicine has highly developed around organizing, preparing, storing and transmitting medical data for a variety of purposes; one of these is to upgrade the human ability to analyze, treat and evaluate pathologic conditions [1]. Data Mining has turned out to be the most frequently used term in medical science and is highly dependent on Data Mining tools and techniques for accurate and on time diagnosis [2]. The use of classification technique is found to be very much facilitative for medical experts in diagnosing diseases and making the process more accurate and fast [3].

Going through a normal period of pregnancy and delivery, and having a healthy baby are common wishes of every mother. Outweighing the fetus status, preparing psychologically and physically, Obstetrician would determine the suitable delivery means in case of any atypical situations according to the diagnostic outcome of the examination then the fetal risk or adverse ending could be minimized or even eliminated [4].

Received: April 19, 2019

Reviewed: June 3, 2019

Accepted: June 6, 2019 
Cesarean section is one of the most common surgical procedures in Gynecology and is considered to be the only option performed in case of fetal distress or any other emergency conditions [5]. Fetal distress usually ends in complications during labor. CTG a well-known technique, also known as electronic fetal monitoring (EFM); consists of simultaneous monitoring of FHR and Uterine contractions (UC) printed on a single stripe of paper[6]. EFM tests are applied to avoid or handle an unwanted situation and keeping the baby and mother safe. Aim of EFM tests is to Information gathered from CTG is used for identification for pathologic fetus state and assist obstetricians to anticipate future complications and interfere before permanent harm happens to the fetus [2]. CTG report is evaluated by an expert clinician using specific guidelines; however, such expertise is not always available making the CTG evaluation a hard task [7].

\section{PROBLEM DEFINATION}

Still, after the introduction of CTG in clinical practice, the predictive power of these methods remains disputable and still erroneous[8]. During CTG's record analysis, clinical decisions are made by visual inspection of CTG traces [5]. However, the main weakness of this approach is poor human interpretations leading to inter- and intra-observer variability [4]. Since the interpretation of the information provided by the CTG is not standardized leading to unnecessary surgical intervention and fetus loss [9]. Complications during labor, long-term disabilities and deaths are still an important cause of suffering for parents and families even in industrialized countries.

Efforts have been carried out over the past three decades for the development of sophisticated automated systems [7]. It has been noticed from the research literature that the use of a classification technique is highly beneficial in terms of time and accuracy. Classification is thus employed to analyze and classify CTG data and facilitate physicians in taking important decisions and avoid human mistakes [11]. Therefore the current study also includes classification technique over CTG data. It has also been noticed that classifiers give glowing results if some preprocessing techniques are used over the dataset before classification. For the reason; a preprocessing technique is known as "Feature Selection" which is used over dataset "CTG" to accelerate the classification performance of classifiers. First the dataset; CTG is preprocessed; eliminated the unnecessary attributes and then the preprocessed dataset is classified using each classifier. The results gathered are evaluated using evaluation metrics and then compared in order to find a classifier that performs best.

The related work of the study is discussed in Section 2. The overview of the classification techniques is presented in Section 3 and the detail of the experimental setup of the study is presented in Section 4. Finally, the study is concluded in Section 5 .

\section{RELATED WORK}

Potharaju [12], used KKN and DT over CTG dataset. To cope with imbalance data, Symmetric Minority Over-Sampling Technique (SMOTE) was used in the study. Fergus [5], in their study, used RF over CTG dataset. They proposed an RF-based decision support system for prediction of caesarean sections and normal deliveries. SVM and RF are compared by Nagendra [6], for evaluating the fetal state prediction. Their study has reduced the feature set by obtaining most important features, Extra tree classifiers, and Logistic Regression; feature ranking approaches were used in the study. For the precise identification of suspicious and pathologic cases, Bhatnagar and Maheshwari [8], used DT, NB, and RF over CTG dataset. The dimensionality of the dataset is reduced using attribute selection. DT, KNN, LR, RF and SVM were used by Sahin and Subasi[13]. Sahin and Subasi evaluated the performance of classification algorithms by using them over CTG dataset. CTG dataset in their case includes two fetal states Normal and 
Pathologic. Shah [14], proposed bagging approach in combination with DT for the classification of CTG data into normal, suspicious and pathologic fetal states. DT based Adaptive Boosting classification system was used by Karabulut and Ibrikci [9], in their study for analyzing CTG data including diagnostic features for discriminating a pathologic fetus. They also used SVM and NB with adaptive boosting but most promising classifier they found was DT. For the classification of CTG data, Georgoulas [7], proposed an ordinal classification approach using DT as the base classifier. They have also used SMOTE technique for data balancing. DT, SVM and NB were used by Spilka [15], for the classification FRH. In their work [15], has combined nonlinear features with conventional features. Polat and Mehr [16] used Correlation-based Feature Selection (CFS) approach along with SVM classifier for the classification of chronic kidney disease. RF-based classification system was proposed for prediction of cardiovascular risk. For selecting a set of relevant features used the CFS approach and obtained 91.6\% accuracy [17]. Singh and Vyas [18], also used CFS in their study and found it favorable. Alyami, investigated the effect of CFS on breast cancer diagnosis system with ANN and SVM classification algorithms. CFS is used in a predictive system for online news popularity. Jain and Vinod [21], proposed CFS based improved-Binary Practical Swarm Optimization system for Gene Selection and cancer classification. Aim of all these researches is to improve the classification accuracy for which they have used different preprocessing techniques. As mentioned earlier Potharaju [12] used data balancing technique, Fergus [5] used the dataset for prediction of normal delivery and caesarean section, Nagendra [6] used feature ranking approach, Shah [14] used Bagging approach, Karabulut and Ibrikci [9] used Adaptive boosting approach, Georgoulas [7] used Ordinal classification approach.

The focus of our study is to enhance the performance of classifiers. As preprocessing a feature selection technique; Correlation-based feature selection is used to extract a subset of highly correlated attributes. The dataset with reduced feature set is classified with classification algorithms then.

\section{CLASSIFICATION}

Classification is the most important one among DM's techniques [22]. Classification is a supervised learning approach having known class labels. The input feature vector is mapped by the classifier into an output class label. Both numerical and categorical values can be handled. Binary and Multilevel are two classification methods. CTG dataset used in this study is based on three FHR states; therefore multilevel classification is being used here. Using multilevel classification, the dataset is classified into three classes: Normal, Suspect and Pathologic.

Classification algorithms used in this study are:

\section{DECISION TREE}

Decision tree (DT) classifier uses a tree-like graph similar to a flowchart where node denotes a test on a particular attribute and the branch denotes an outcome of the test whereas the class labels are represented by the leaves [23] A DT considers all possible tests that can split the data and chooses the one that gives highest information gain [7].

Generally, a DT is construction is based on 2 phases:

- A "Recursive" phase: top-down process, grows a tree to fit the training data

- A "Pruning" phase: to pass up over-fitting

For any kind of problem, DT doesn't require any domain knowledge [24]. The extreme usage of DT is in process research analysis for computing the conditional probability. DT is easy to understand, handle huge dataset, and simply interpret and robust as well as it knobs both categorical and numerical data[25]. 


\section{K-NEAREST NEIGHBOR}

K-Nearest Neighbor (KNN) is a member of lazy learning algorithms' family and is considered the simplest of all classifiers [24]. Since KNN is an instance based on a classifier, it can easily be implemented in parallel. KNN uses majority voting among the labels for $\mathrm{K}$ closest data points in feature space; where $\mathrm{K}$ is an integer value. The closest data point is determined using: Euclidean distance as a distance measure for continuous variables and Hamming distance as a distance measure for discrete variables [23]. KNN classifier is also known as case-based reasoning and has been used in many applications like recognition and estimation. Due to its simplest nature and high convergence speed, it is preferred over other classifiers [27].

\section{LOGISTIC REGRESSION}

Logic Regression (LR) is a type of nonlinear regression and accepts categorical data as input. The idea of LR was introduced by Cox in 1958, with the principle of estimating a binary response based on a set of independent features. LR takes input feature set and then predicts the probability of occurrence using the logistic function [23].

\section{SUPPORT VECTOR MACHINES}

The idea of SVM was first proposed by Vladimir Vapnik and Cortes in 1958 within the area of statistical theory and structural risk minimization for the purpose of classification and regression [28]. The basic idea of SVM is to have maximum margins between different samples; arranging the margins of the samples such that they got separated by maximum distance [1]. The data points are separated by hyper-planes and there exist an infinite number of hyper-planes. SVM can find the optimum disjunctive hyper-plan with maximum margins [16]. SVM has been used for solving problems like pattern recognition and regression estimation. SVM has also shown promising results when applied in the medical domain for diagnosis of disease classification [1].

\section{RANDOM FOREST}

Random Forest (RF) was introduced by Breiman in 2001. RF is basically a combination of two methods: random space method and bagging decision trees. RF classifier contains multiple DTs which are trained according to the bagging method. The final classification result is obtained after getting the result of all DTs and voting on the result of all DTs. For coping with issues like Over-fitting and Under-fitting, RF is extremely suggested classifier. RF also has the capability to handle noise and outliers. RF is a very popular classification algorithm and has been applied with success in the classification of different kinds of medical datasets [30].

\section{NAÏVE BAYES}

Naive Bayes (NB) is a statistical classifier and does classification based on probabilities and has the advantages of accurate classification and high computational efficiency. NB is preferred when the dimensionality of the input data is high. In NB based classification, the label that maximizes the posterior probability is returned as output [22]. NB performs classification for given input $X$ as:

Let $\mathrm{T}$ be a training set and there be $\mathrm{m}$ number of classes $\mathrm{C}_{1}, \mathrm{C}_{2}, \mathrm{C}_{3} \ldots \mathrm{Cm}$. Each tuple of the training set is represented by an n-dimensional attribute: $A_{1}, A_{2}, A_{3} \ldots A_{n}$. NB will predict that $\mathrm{X}$ belongs to the class $\mathrm{C}_{\mathrm{i}}$ if:

$$
P\left(C_{i} / X\right)>P\left(C_{j} / X\right) \text { for } 1 \leq j \leq m ; \mathrm{i} \neq \mathrm{j}
$$


This concept is known as the Prior Probability or Posterior Probability. So a new input will fall into the class having the highest Prior Probability. Naïve Bayes has the advantages of accurate classification and high computational efficiency.

\section{DATASET AND EXPERIMENT}

\section{A. CTG DATASET}

The dataset used in this study is from the University of California Irvin (UCI) machine learning repository. CTG dataset is the source of information necessary for the analysis and creation of fetal health predictive model[13]. It also provides information about fetus health status that helps for early intervention before the risk occurrence. CTG contains measurements of FHR and uterine contractions features on cardiotocogram; defined by 23 attributes and has 2126 instances classified as three fetal states: 1655 normal, 296 suspects and 176 pathologic cases. The records contained were automatically processed and the respective diagnostic features measured. The cardiotocograms were classified by expert obstetricians and a consensus classification label assigned to each of them. Attributes of the dataset are described in Table I.

For being used in this study, the dataset is broken up into training and testing set where the training set holds $80 \%$ of the entire dataset and the rest $20 \%$ is included in the testing set.

\section{B. METHODOLOGY}

The study is conducted following two-steps methodology. The first step of the used methodology is dataset collection. The collected data is then preprocessed in the second step. The preprocessing step has two sub-steps: Feature selection and Normalization. The dataset is preprocessed to make the classification result more accurate. In third step, the dataset is divided into training and testing sets. The fourth step is the classification; each classification algorithm is applied over the training set, a classification model is constructed. The constructed model is tested using the testing set. Results for each classification model is collected and compared. Figure 1 shows the flow chart of the methodology used in this study.

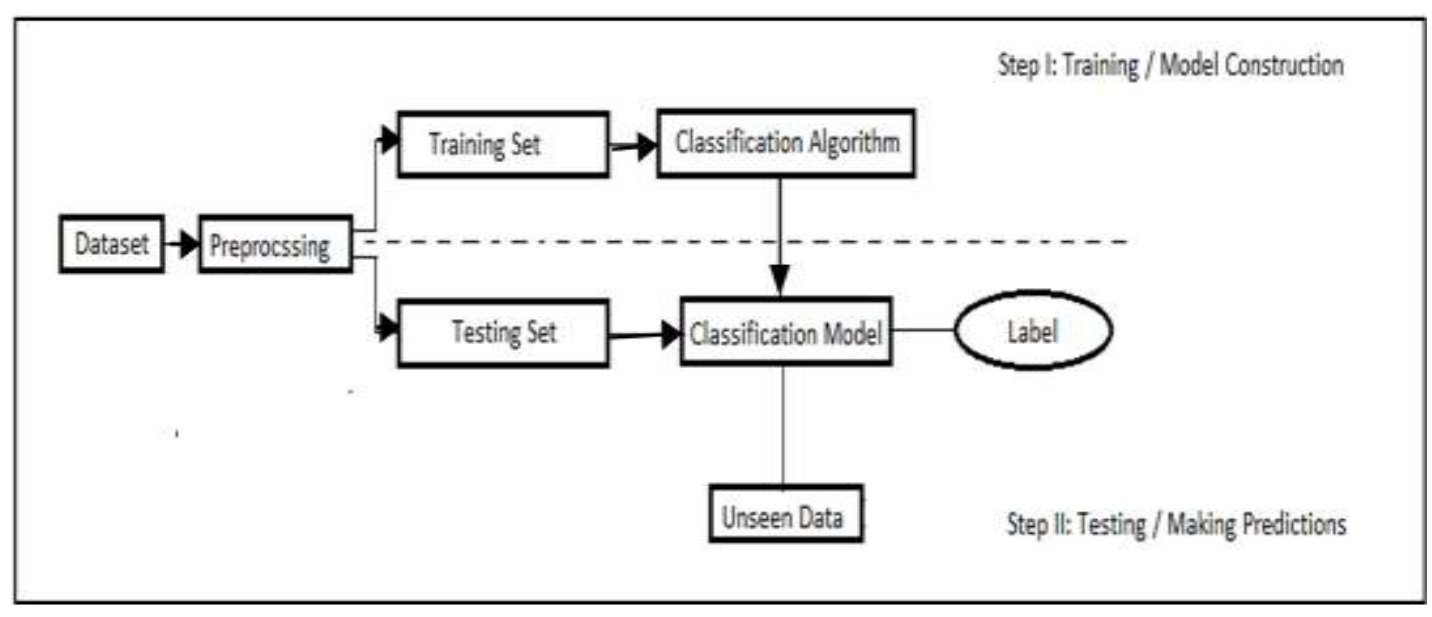

Fig. 1 Methodology's Flowchart 
Table I. CTG Attributes and their Description

\begin{tabular}{|l|l|}
\hline \multicolumn{2}{|c|}{ Attributes and Descriptions } \\
\hline LB & FHR Baseline (bmp) \\
\hline AC & Number of Accelerations per Second \\
\hline FM & Number of Fetal movements per seconds \\
\hline UC & Number of Uterine Contractions per second \\
\hline DL & Number of Light Decelerations per second \\
\hline DS & Number of Severe Decelerations per second \\
\hline DP & Number of Prolonged Decelerations per seconds \\
\hline ASTV & Percentage of time with Abnormal Short Term Variability \\
\hline MSTV & Mean value of Short Term Variability \\
\hline ALTV & Percentage of Time with Abnormal Long Term Variability \\
\hline MLTV & Mean value of Long term Variability \\
\hline Width & Width of FHR histogram \\
\hline Min & Minimum of FHR histogram \\
\hline Max & Maximum of FHR histogram \\
\hline Nmax & Number of histogram peaks \\
\hline Nzeros & Number of histogram zeros \\
\hline Mode & Histogram Mode \\
\hline Mean & Histogram Mean \\
\hline Median & Histogram Median \\
\hline Variance & Fetal State (N: normal, S: suspect, P: pathologic) \\
\hline Tendency & \\
\hline NSP & Histogram Tendency \\
\hline
\end{tabular}

\section{PRE-PROCESSING}

Before carrying out the experiment, the raw data needs to be preprocessed to convert it into something useful [2]. Preprocessing here is based on two steps:

1) Feature Selection: To accelerate the classification performance of the classifiers, a feature selection technique, Correlation-based feature selection is used. The unnecessary (less correlated) attributes are neglected and experiment is conducted considering the highly correlated attributes. Highly correlated attributes are those that have a high positive and high negative correlation with the class attribute.

A tool called "RapidMiner Studio" is used to find the correlation among the attributes. RapidMiner gives a "Correlation Matrix" showing the correlation of each attribute with the class attribute; NSP. The attributes with least positive and least negative correlation values; $\mathrm{DS}=\mathrm{NaN}, \mathrm{LB}=0.14851, \mathrm{FM}=0.087933, \mathrm{Min}=0.063175, \mathrm{DL}=0.062702$ and Nzeros $=-0.01668$ are excluded and the rest are selected to be used in the experiment. Table II shows the correlation of attributes with a class attribute.

2) Normalize: Normalization is the second phase of preprocessing. Data is normalized to rescale attributes to the range of 0 to 1 where 0 indicates the least and 1 indicates the most. In this step, a normalize filter in WEKA (version 8.3.1) is used over the data. 
Table II. Correlation between each attribute and the class attribute

\begin{tabular}{|l|l|}
\hline \multicolumn{1}{|c|}{ Attribute } & \multicolumn{1}{|c|}{ Correlation with NSP } \\
\hline LB & 0.148151 \\
\hline AC & -0.36385 \\
\hline FM & 0.087933 \\
\hline UC & -0.20382 \\
\hline DL & 0.062702 \\
\hline DS & NaN \\
\hline DP & 0.488277 \\
\hline ASTV & 0.471191 \\
\hline MSTV & -0.10338 \\
\hline ALTV & 0.426146 \\
\hline MLTV & -0.2268 \\
\hline Width & -0.06879 \\
\hline Min & 0.063175 \\
\hline Max & -0.04527 \\
\hline Nmax & -0.02367 \\
\hline Nzeros & -0.01668 \\
\hline Mode & -0.25041 \\
\hline Mean & -0.22699 \\
\hline Median & -0.20503 \\
\hline Variance & 0.20663 \\
\hline Tendency & -0.13198 \\
\hline & \\
\hline
\end{tabular}

\section{EVALUATION METRICS}

To evaluate the classification performance of the classifiers four evaluation metrics are used:

Accuracy: The ratio of correctly classified instances to the total number of instances. Accuracy is calculated as:

$$
\text { Accuracy }=\frac{T P+T N}{T P+F P+T N+F N}
$$

Precision: The ratio of correct positive predictions to the total number of positive predictions made.

$$
\text { Precision }=\frac{T P}{T P+F P}
$$

Recall: The ratio of correct positive predictions made to the total number of actual positive instances. Recall is calculated as:

$$
\text { Recall }=\frac{T P}{T P+F N}
$$


F-measure: Average of Precision and Recall. It takes both FPs and FNs into account and is calculated as:

$$
F-\text { measure }=2 * \frac{\text { Precision }^{*} \text { Recall }}{\text { Precision }+ \text { Recall }}
$$

\section{E. EXPERIMENT}

WEKA 3.8.1 is used for carrying out experiments. Two experiments were carried out. Each classifier is applied individually on the training set to build a classification model. The created classification model is then used to classify the test set. This process of building a classification model and then testing it is repeated for each individual classifier. The experimental result is shown in Table II and Table III. Experiment I is carried over a dataset including the complete feature set.

Table III. Result of Experiment I

\begin{tabular}{|l|c|c|c|c|}
\hline \multicolumn{1}{|c|}{ Classifier } & Accuracy & Precision & Recall & F-measure \\
\hline J48 & $67.06 \%$ & $84.10 \%$ & $67.10 \%$ & $72.00 \%$ \\
\hline IBK & $79.76 \%$ & $84.70 \%$ & $79.80 \%$ & $81.10 \%$ \\
\hline Logistic & $65.88 \%$ & $91.70 \%$ & $65.90 \%$ & $75.10 \%$ \\
\hline SMO & $67.53 \%$ & $83.80 \%$ & $67.50 \%$ & $67.90 \%$ \\
\hline RandomForest & $78.12 \%$ & $63.60 \%$ & $78.10 \%$ & $70.10 \%$ \\
\hline NaiveBayes & $81.18 \%$ & $89.30 \%$ & $81.20 \%$ & $84.40 \%$ \\
\hline
\end{tabular}

Experiment II is carried over a dataset including a feature set of highly correlated attributes; choosing a feature subset of 16 high correlated attributes and eliminating the 6 attributes, less correlated to the class attribute. Aim of this feature selection is improving the classifiers' performance. The impact of Correlation-based Feature Selection can be seen in Table IV.

Table IV. Result of Experiment II

\begin{tabular}{|l|c|c|c|c|}
\hline \multicolumn{1}{|c|}{ Classifier } & Accuracy & Precision & Recall & F-measure \\
\hline J48 & $69.18 \%$ & $77.10 \%$ & $69.20 \%$ & $69.50 \%$ \\
\hline IBK & $79.06 \%$ & $85.40 \%$ & $79.10 \%$ & $80.60 \%$ \\
\hline Logistic & $73.41 \%$ & $92.40 \%$ & $73.40 \%$ & $79.20 \%$ \\
\hline SMO & $70.59 \%$ & $84.10 \%$ & $70.60 \%$ & $70.10 \%$ \\
\hline RandomForest & $77.65 \%$ & $63.70 \%$ & $77.60 \%$ & $69.90 \%$ \\
\hline NaiveBayes & $85.88 \%$ & $94.60 \%$ & $85.90 \%$ & $89.50 \%$ \\
\hline
\end{tabular}

\section{F. RESULT}

Table II and Table III present the results obtained from the experiment I and II respectively. Since the aim of our study was to enhance the performance of classifiers. According to research literature we can enhance the classification result by the reducing feature set A preprocessing technique Correlation-based Feature selection is used in this study for reducing the feature set such that the features which are less important and have less impression on the class attribute are excluded and including the attributes which are having powerful impression on the class attribute. The impact of feature selection can be seen by comparing the results of the experiment I and II.

It can be noticed that there is an improvement in the performance of the classifiers with a reduced feature set. With respect to accuracy; j48, Logistic, SMO and Naïve Bayes have improved by $2.12 \%, 7.53 \%, 3.06 \%$, and $4.7 \%$ respectively whereas KNN's and Random Forest's accuracy is decreased by $0.7 \%$ and $0.47 \%$ respectively. In terms of Precision; KNN, Logistic, SMO, Random Forest and Naïve Bayes have improved by $0.7 \%, 0.7 \%, 0.3 \%, 0.1 \%$ and $5.3 \%$ respectively while $\mathrm{j} 48$ 's performance is reduced by 
$0.7 \%$. In terms of Recall; j48, Logistic, SMO and Naïve Bayes have improved by $2.1 \%$, $7.5 \%, 3.1 \%$ and $4.7 \%$ respectively while KNN's and Random Forest's recall falls by $0.7 \%$ and $0.5 \%$ respectively. Similarly, in terms of F-measure; Logistic, SMO and Naïve Bayes have improved by $4.1 \%, 2.2 \%, 3.06 \%$ and $5.1 \%$ respectively. KNN, j48 and Random Forest's values for F-measure are reduced by $0.5 \%, 2.5 \%$ and $0.2 \%$ respectively.

Closing the result, it has been noticed that the overall outcome of using Correlationbased feature selection is fruitful. Performance of all used classifiers has been enhanced using the preprocessing technique except KNN and RandomForest. But in this specific situation, more promising results were obtained by Naïve Bayes by achieving $85.88 \%$ accuracy, $94.60 \%$ precision, $85.90 \%$ recall and $89.50 \%$ f-measure.

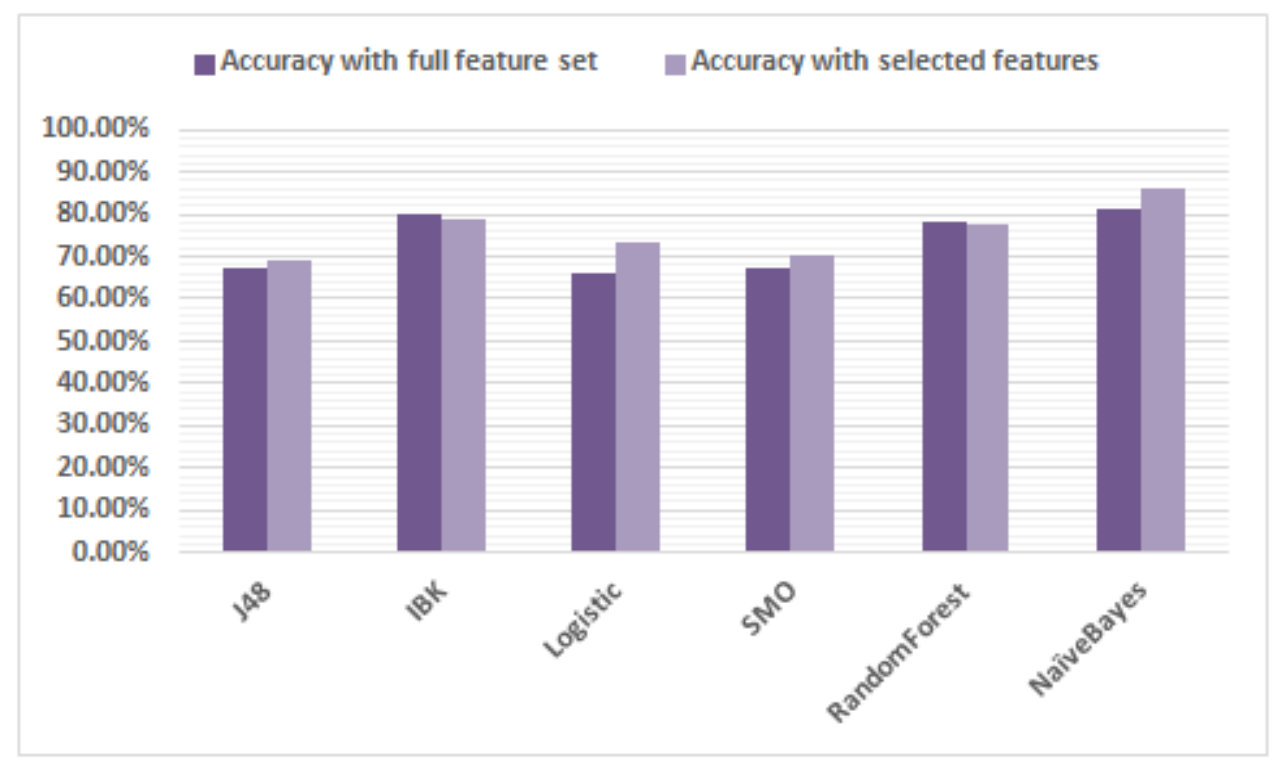

Fig. 2 Accuracy Comparison of Classification Techniques

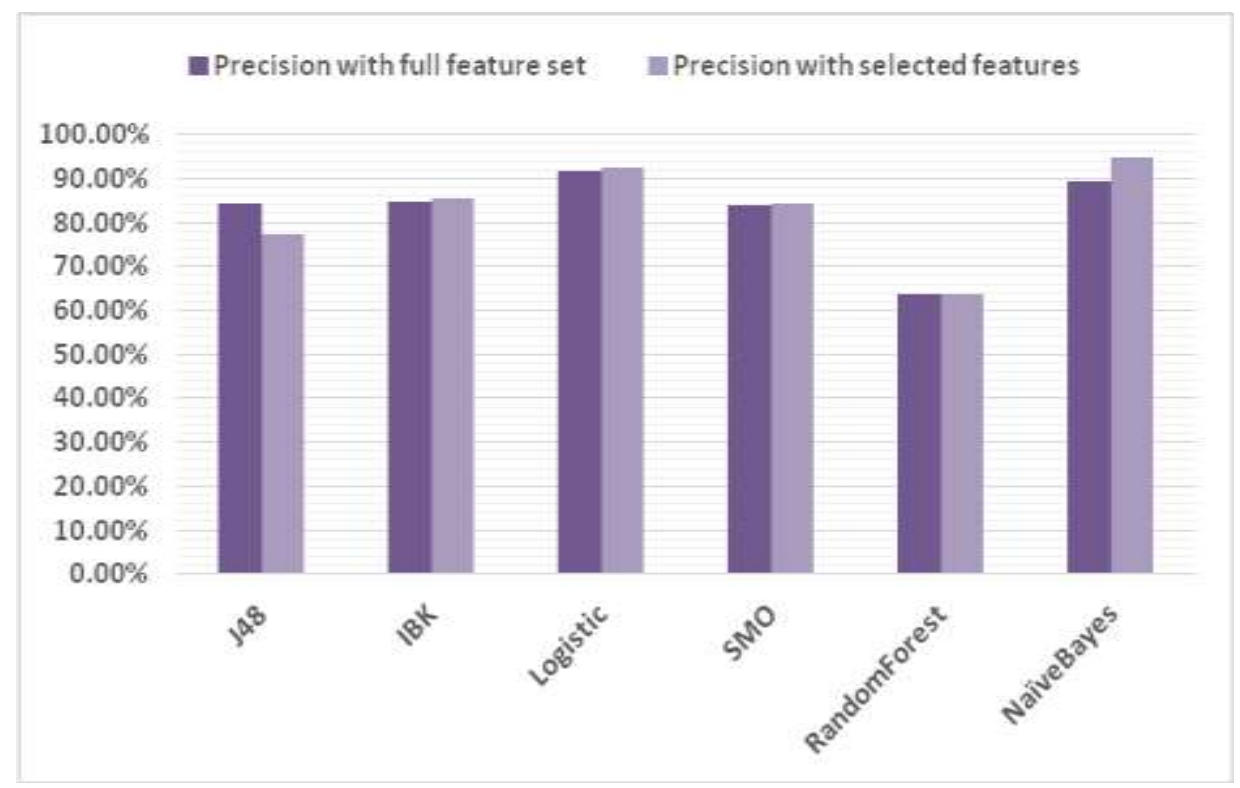

Fig. 3 Precision Comparison of Classification Techniques 


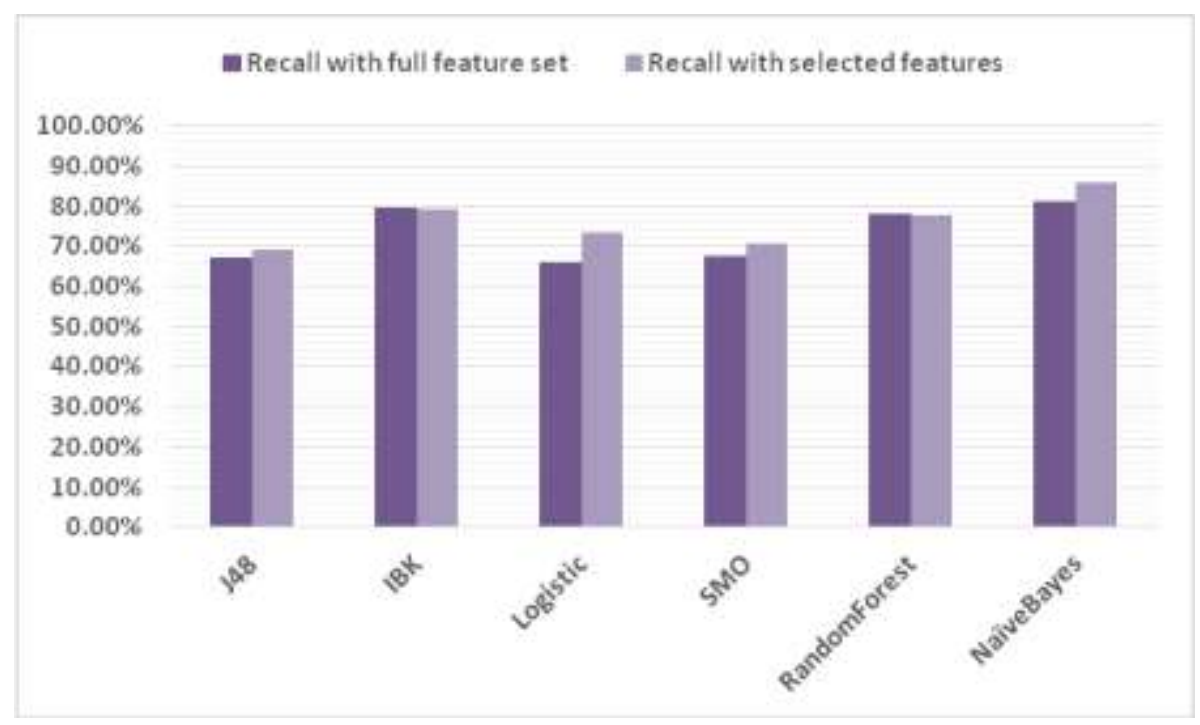

Fig. 4 Recall Comparison of Classification Techniques

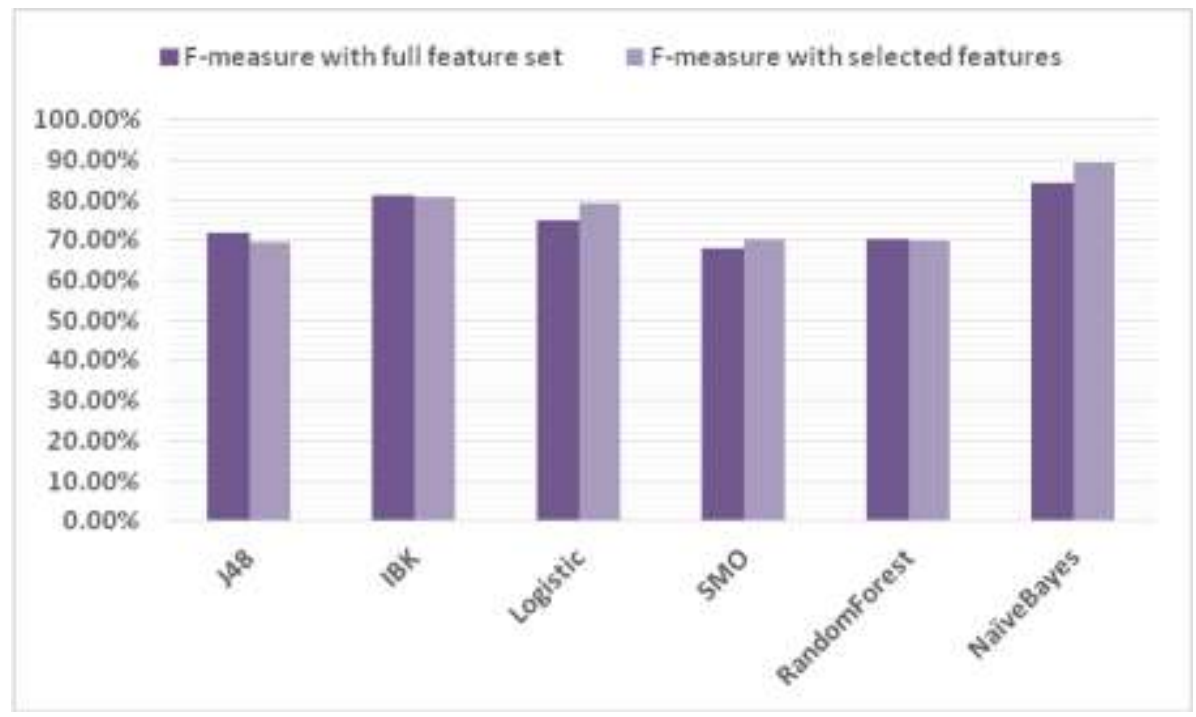

Fig. 5 F-measure Comparison of Classification Techniques

\section{CONCLUSION}

In this study, the focus remained on the application of classification technique in Gynecology and Obstetrics for the classification of CTG data in order to predict the normal, suspect and pathologic cases. Six well-known classification algorithms are evaluated for classification of fetal state in CTG dataset. Aim of our study was to enhance the classification performance of classifiers. The research literature provides proof for the advantageous nature of Correlation-based feature selection technique. So to achieve our goal, we have used the same preprocessing technique for reducing the feature set; Correlation based Feature Selection over CTG dataset. First, we have obtained the correlation values of each attribute to the class attribute. Next, we eliminated the attributes that have less value of correlation with the class attribute. For analyzing the positive impact of used feature selection technique, we conducted two experiments over dataset one with the full feature set and second with a reduced feature set; considering the feature set of highly correlated attributes. By gathering the results of both experiments, it has been noticed that the overall outcome of using Correlation-based feature selection is bountiful. Performance of all used classifiers has been enhanced using the preprocessing 
technique except KNN and Random Forest. But in this specific situation, more promising results were obtained by Naïve Bayes by achieving $85.88 \%$ accuracy, $94.60 \%$ precision, $85.90 \%$ recall and $89.50 \%$ f-measure.

This study would be really helpful in descending any unwanted condition during labor that became a threat to baby's and/or mother's life and to protect the families facing sorrowful situations. Since the mentioned technique can predict the fetus's health state by monitoring the FHR. In the case of sensitive situations, the obstetricians would take preventive steps for the safety of the baby's life.

In a nutshell, numerous researchers have used classification technique in their work. Researchers have proposed and used scores of classification algorithms in different scenarios including medicine. More specifically in medicine, use of classification can help the physicians for on-time and accurately diagnose, since classification is a quick and less expensive approach.

\section{REFERENCES}

[1] Hasan, Tabreer T., Manal H. Jasim, and Ivan A. Hashim. "Heart Disease Diagnosis System based on Multi-Layer Perceptron neural network and Support Vector Machine." (2017).

[2] Khanna, Dishant, and Arunima Sharma. "Kernel-Based Naive Bayes Classifier for Medical Predictions." Intelligent Engineering Informatics. Springer, Singapore, 2018. 91-101.

[3] Warmerdam, G. J. J., et al. "Detection rate of fetal distress using contraction-dependent fetal heart rate variability analysis." Physiological measurement 39.2 (2018): 025008.

[4] Zhang, Yang, and Zhidong Zhao. "Fetal state assessment based on cardiotocography parameters using PCA and AdaBoost." 2017 10th International Congress on Image and Signal Processing, BioMedical Engineering and Informatics (CISP-BMEI). IEEE, 2017.

[5] Fergus, Paul, et al. "Classification of caesarean section and normal vaginal deliveries using foetal heart rate signals and advanced machine learning algorithms." Biomedical engineering online 16.1 (2017): 89.

[6] Nagendra, Vinayaka, et al. "Evaluation of support vector machines and random forest classifiers in a real-time fetal monitoring system based on cardiotocography data." 2017 IEEE Conference on Computational Intelligence in Bioinformatics and Computational Biology (CIBCB). IEEE, 2017.

[7] Georgoulas, George, et al. "An ordinal classification approach for CTG categorization." 2017 39th Annual International Conference of the IEEE Engineering in Medicine and Biology Society (EMBC). IEEE, 2017.

[8] Bhatnagar, Divya, and Piyush Maheshwari. "Classification of cardiotocography data with WEKA." International Journal of Computer Science and Network-IJCSN 5.2 (2016).

[9] Karabulut, Esra Mahsereci, and Turgay Ibrikci. "Analysis of cardiotocogram data for fetal distress determination by decision tree based adaptive boosting approach." Journal of Computer and Communications 2.09 (2014): 32.

[10] Signorini, Maria G., and Giovanni Magenes. "Advanced signal processing techniques for CTG analysis." XIV Mediterranean Conference on Medical and Biological Engineering and Computing 2016. Springer, Cham, 2016.

[11] Potharaju, Sai Prasad, et al. "Data mining approach for accelerating the classification accuracy of cardiotocography." Clinical Epidemiology and Global Health (2018).

[12] Sahin, Hakan, and Abdulhamit Subasi. "Classification of the cardiotocogram data for anticipation of fetal risks using machine learning techniques." Applied Soft Computing 33 (2015): 231-238.

[13] Shah, Syed Ahsin Ali, et al. "Decision trees based classification of cardiotocograms using bagging approach." 2015 13th International Conference on Frontiers of Information Technology (FIT). IEEE, 2015.

[14] Spilka, Jirí, et al. "Using nonlinear features for fetal heart rate classification." Biomedical Signal Processing and Control 7.4 (2012): 350-357.

[15] Polat, Huseyin, Homay Danaei Mehr, and Aydin Cetin. "Diagnosis of chronic kidney disease based on support vector machine by feature selection methods." Journal of medical systems 41.4 (2017): 55.

[16] Xu, Shan, et al. "Cardiovascular risk prediction method based on CFS subset evaluation and random forest classification framework." 2017 IEEE 2nd International Conference on Big Data Analysis (ICBDA)(. IEEE, 2017.

[17] Singh, Bharat, and Om Prakash Vyas. "Maximum Spanning Tree Based Redundancy Elimination for Feature Selection of High Dimensional Data." International Arab Journal of Information Technology 15.5 (2018): 831-841.

[18] Jain, Indu, Vinod Kumar Jain, and Renu Jain. "Correlation feature selection based improved-binary particle swarm optimization for gene selection and cancer classification." Applied Soft Computing 62 (2018): 203-215. 
[19] Gorade, M. S. M., A. Deo, and P. Purohit. "A study of some data mining classification techniques." IRJET 4 (2017): 3112-3115.

[20] Dadar, Mahsa, et al. "Performance comparison of 10 different classification techniques in segmenting white matter hyperintensities in aging." NeuroImage 157 (2017): 233-249.

[21] Tomar, Divya, and Sonali Agarwal. "A survey on Data Mining approaches for Healthcare." International Journal of Bio-Science and Bio-Technology 5.5 (2013): 241-266.

[22] Aziz, Abdul, and Aziz Ur Rehman. "Detection of Cardiac Disease using Data Mining Classification Techniques." International Journal of Advanced Computer Science and Applications 8.7 (2017).

[23] Jabbar, M. A. "Prediction of heart disease using k-nearest neighbor and particle swarm optimization." Biomed. Res 28.9 (2017): 4154-4158.

[24] Yahiaoui, Amani, Orhan Er, and Nejat Yumusak. "A new method of automatic recognition for tuberculosis disease diagnosis using support vector machines." Biomed. Res 28 (2017): 4208-4212.

[25] Le Douget, Jean-Eudes, et al. "Surface and intracranial EEG spike detection based on discrete wavelet decomposition and random forest classification." 2017 39th Annual International Conference of the IEEE Engineering in Medicine and Biology Society (EMBC). IEEE, 2017. 